\title{
Practice and Thinking of Traditional Chinese Medicine Agriculture Helping Rural Revitalization
}

\author{
Lijian Zhang* \\ Biotechnology Research Institute, Chinese Academy of Agricultural Sciences, Beijing, China
}

\begin{tabular}{l} 
ARTICLE INFO \\
Article history \\
Received: 16 June 2021 \\
Accepted: 1 July 2021 \\
Published Online: 31 July 2021 \\
\hline
\end{tabular}

Keywords:

Traditional Chinese Medicine (TCM)

Agriculture

Rural

\section{Introduction}

Traditional Chinese medicine is a scientific system based on ancient Chinese materialism and dialectics as the philosophy and the holism as the guiding ideology. Traditional Chinese medicine understands nature, and life with a connected, developing and comprehensive perspective. What the "Traditional Chinese Medicine Agriculture" means is to apply the principles and methods of traditional Chinese medicine to the agricultural field, and to realize the cross-border integration of modern agriculture and traditional Chinese medicine, complementing each other's advantages and integrated innovation.

"Traditional Chinese medicine agriculture" is the carrier of Chinese traditional farming culture, the crystallization of the diligence and wisdom of the Chinese nation for thousands of years, the integrated inheritance and innovative development of agricultural culture and traditional Chinese medicine culture, and an ecological agriculture

\section{ABSTRACT}

China is a big agricultural country with a long history, and has created a brilliant agricultural civilization. Agriculture has always been an important foundation of China's national economy and has made great achievements. At present, promoting sustainable agricultural development has become an important part of the strategic adjustment of China's economic development mode, and it is of great significance to explore and strive to blaze a path of sustainable agricultural development with Chinese characteristics.

with Chinese characteristics. "Traditional Chinese medicine agriculture" keeps pace with the times and has made rapid progress in the field of research and development. It has played an important role in production practice and will make due contributions to "rural revitalization" and "the construction of a community with a shared future for mankind".

"Traditional Chinese medicine agriculture" can be the comprehensive prevention and control of water, soil and gas pollution and improve the producing environment, promote the healthy growth of animals and plants, ensure the effective supply and quality and safety of agricultural products, and explore a new way for the sustainable development of agriculture in China and even the world. "Traditional Chinese medicine agriculture" is an ecological agriculture with Chinese characteristics.

"Traditional Chinese medicine agriculture" has three characteristics: first it is systematic, focusing on the internal connection of agricultural ecosystem and all parts of

About the Author:

Lijian Zhang,

Former vice president of Chinese Academy of Agricultural Sciences, "Traditional Chinese Medicine Agriculture” initiative scientist, Researcher, Doctoral Supervisor;

Biotechnology Research Institute, Chinese Academy of Agricultural Sciences, Beijing, China; 
the organism, which is the essential requirement of maintaining relative stability and harmony between agricultural components; second, it is comprehensive, forming multi-faceted, multi-level composite effect, namely achieving comprehensive effect through comprehensive means; third, it is whole, emphasizing covering all production units and planting and breeding chain.

\section{The Theory and Practice of "Traditional Chinese Medicine Agriculture"}

It is mainly reflected in three aspects: 1 , producing pesticide and veterinary medicine to protect the growth of animals and plants; 2, producing fertilizers and feed through the combination of Chinese herbal medicine and natural nutrition elements to promote the growth of animals and plants; 3 , regulating the growth of animals and plants by the mutual promotion and restrict mechanism between animals and plants (such as live Chinese herbal medicine organism) and other biological communities.

"Traditional Chinese medicine agriculture" can reduce the use of chemical pesticides, chemical fertilizers, chemical feed additives and various antibiotics and hormones, which is conducive to the healthy growth of animals and plants, and the implementation of environment-friendly prevention and control of diseases and insect pests.

"Traditional Chinese medicine agriculture" keeps pace with the times and has had a good impact at home and abroad. It has made rapid progress in the research and development, and has played an important role in production practice. CNKI journal paper search results show more than 6000 papers about "TCM agriculture", and there are hundreds of thousands of researchers in "TCM agriculture" related research and development projects in national universities, scientific research agencies, and more than 1 million agricultural operators apply "TCM agriculture" thinking and techniques in agricultural production progresses, with good results, and they have accumulated a lot of experience and data. At the same time, developing a large number of "TCM agriculture" series of inputs.

In recent years, various inputs of "TCM agriculture" have shown remarkable results in production practice. Take three of the inputs of TCM agricultural series as an example: in 2019, 34 agricultural products of 520,000 mu in 26 provinces (cities and autonomous regions), generally manifested as high quality and high yield, ecological safety, integrity, full color and fragrance, strong functionality, long preservation period, good adverse resistance and reduced production costs. At the same time, the soil repair agent of TCM agriculture input can significantly improve the soil mass structure, microbial community and organic matter content.
At present, in the production practice, "TCM agriculture" has formed a 10 series of inputs with good results. In terms of market development, agricultural production experiments show that the agricultural products produced by TCM agricultural technology have a clear degree of product quality differentiation. The concept of agricultural products in traditional Chinese medicine is easier to be accepted by consumers. Traditional Chinese medicine agricultural products have the characteristics of "differentiation", which will help to build a well-known local agricultural products brand. "Traditional Chinese medicine agriculture" can produce "food homologous medicine" or "functional agricultural products" that are conducive to the sub-health population accounting for about $70 \%$ of the total population to improve their immunity, which will produce important application value in the development and application of food homologous medicine, health food and food for special medical use.

"Traditional Chinese medicine agriculture" has innovated the way of agricultural scientific research and production methods, providing new and huge space for research and development for the innovation of agricultural science and technology system with Chinese characteristics, and "Traditional Chinese medicine agriculture" has created a new field to explore the sustainable development of mankind and the world.

In practice, we can connect with industrial strong town projects. "Traditional Chinese Medicine Agricultural Industry Strong Town Project" are composed of four levels of business formats: 1, Basic business format, which generally refers to the basic agriculture (traditional Chinese medicine agricultural planting, breeding), and processing industry. 2, Intermediate business format, which refers to the business format that has interactive experience with consumers, including catering, leisure, entertainment, health preservation, and cultural experience, etc. 3, Advanced business format, which refers to the TCM agricultural products experience (stores), (monopoly) stores or (characteristic) products that are branded and can be exported. 4, characteristic business format, which refers to the unique local, or the most well-known business format system (authentic traditional Chinese medicinal materials and the local superior TCM agricultural products), etc. The business formats at the four levels are integrated with each other to jointly form the industrial ecosystem of "the towns characterized by TCM agriculture." Experts pointed out that the "Traditional Chinese Medicine Agricultural Industry Strong Town Project" is the way combining urbanization with industry, which not only provides an innovative model for the coordinated and sustainable development of social, economic and ecological, but also provides 
a personalized choice plan for human development. "TCM Agricultural Industry Strong Town Project" is a future community in life. Around the high-end characteristic TCM agricultural industry, the town will gather a number of relevant high-end talents, and form a future community with life, production, ecology and characteristic cultural connotation and spirit. The town will also attract tourists by the uniqueness of the TCM industry, culture and life, deeply integrating the development of traditional Chinese medicine agricultural industry with culture, tourism, health and other industries, to form an emerging agricultural industry chain such as leisure agriculture.

The preparation of "Traditional Chinese Medicine Agricultural Industry Strong Town Project" should establish "TCM agriculture comprehensive demonstration base". Taking the "TCM agricultural planting stereoscopic production (experimental) demonstration base" project as an example, according to the "agricultural application of traditional Chinese medicine principles and techniques (TCM agriculture)" thinking, the "demonstration base" has three objectives: 1. to build an efficient ecological agriculture model with Chinese characteristics; 2 . to produce food homologous medicines and functional agricultural products; 3. to restore and improve the property of authentic traditional Chinese medicinal materials and Chinese medicinal herbs. "TCM agriculture comprehensive demonstration base" should include three levels of design content: 1 , basic level, which optimizes the practice of agricultural production with traditional Chinese medicine technology (producing pesticide and veterinary medicine, feed fertilizer and natural conditioners based on the principle of Chinese herbal medicine blending); 2, intermediate level, which uses traditional Chinese medicine thinking and methods to coordinate agricultural production ideas (based on the mutual promotion and restrict mechanism, use the interaction between biological communities to improve the function of the agricultural system); 3, advanced level, which refers to "holistic view of traditional Chinese medicine" to improve the layout of agricultural production (unity of nature and man, harmony between man and nature, etc.), and integrates the ecological circulation planting and breeding technology mode based on the healthy circulation theory of traditional Chinese medicine.

The basic conditions for the "demonstration base": 1 . The areas with good ecological environment are selected as the project areas; local authentic Chinese herbal medicine and advantageously functional agricultural products and the "TCM agricultural inputs" that have good performance in production practice are selected as pesticide, veterinary drugs, fertilizer, feed and growth regulation; 2 , food homologous medicine or functional agricultural products aimed at improving the sub-health population accounting for about $70 \%$ of the total population are produced, with the local advantage of food and drug homologous agricultural products brand being formed; 3 , the industrial chains are optimized and extended, with an industrial cluster with good benefits and reasonable structure being formed, and the "Internet" in ten places (the relevant big cities) and the sales channels of the TCM agricultural product experience stores being established; 4 , the combination of forest and animal husbandry, efficient planting, characteristic breeding and deep processing are formed, with entrepreneurial innovation, population of science and leisure, science and technology incubation and other functions, and I, II and III industries are intergraded to form the "traditional Chinese medicine agricultural planting and breeding three-dimensional production demonstration base" with a complete industrial chain.

We should use the principle of traditional Chinese medicine holistic view guiding "traditional Chinese medicine agricultural demonstration base" design: 1, agriculture is an integrated management system, covering farming, forest, animal husbandry and fishing, and we should pay attention to the overall function of the agricultural system, according to the local situation to conduct appropriate planting or breeding and the matching with large ecosystem, aiming at the highest overall output level (output, quality and efficiency). 2. According to the regional natural conditions, we should flexibly choose the agricultural planting and breeding structure, building a composite ecological system mode, to benefit the multi-level utilization of material and energy, improving the utilization rate of space and light energy and increasing the quality and yield. 3. Moderate-scale planting and breeding combination can play the ecological function of the holistic agriculture in order to achieve the best output benefits. High efficiency of material circulation and energy conversion must also be built on a certain scale. In addition, a large agricultural system and subsystems with certain scales are needed to effectively resist large natural disasters.4. The diversity of species in the composite ecosystem can improve the efficiency of material circulation and energy conversion, thus can make full use of organic residues, increase the organic source of breeding feed and planting fertilizer, reduce the use of chemical fertilizers and feed additives, and provide natural conditions for pest prevention and control, thus reducing the use of chemical agents.

"Traditional Chinese medicine agriculture" works in the long term, not in the moment, it should accelerate the development on the basis of good security and stability. "Traditional Chinese medicine agriculture" will produce the effect of gathering resources, complementing each 
other's advantages, integrating innovation and win-win cooperation. It has very important social, economic and ecological benefits and will make due contributions to the rural revitalization and healthy China strategy.

\section{3. "Traditional Chinese Medicine Agriculture" Recognized Highly at Home and Abroad}

Former Director-General of the UN Food and Agriculture Organization (FAO) Graziano delivered a speech at the 15th World Congress on Traditional Chinese Medicine and said that the latest concept of "TCM agriculture" will be included in the FAO work plan. "Traditional Chinese medicine originated in ancient Chinese farming society, traditional Chinese medicine and agriculture are the two oldest industries in China. The tradition and modernity of traditional Chinese medicine agriculture get integrated, develop innovatively, and this will be China's major contribution to mankind". Said professor He Jialun, FAO pharmaceutical consultant and vice chairman of the World Federation of Traditional Chinese Medicine Societies. Sang Binsheng, vice chairman and secretary general of the World Federation of Societies of Traditional Chinese Medicine, noted that the agricultural application of traditional Chinese medicine principles and techniques (TCM agriculture) highlights the world. The cross-border integration, complementary advantages and integrated innovation of traditional Chinese medicine and agriculture have far-reaching significance for the development of the two fields.

On November 17, 2018, the 15th World Congress of Traditional Chinese Medicine opened in Rome. Dr. Zhang Lijian, the initiator of agricultural application of traditional Chinese medicine principles and techniques (TCM agriculture), was invited to make the report on the theme of "Developing Traditional Chinese Medicine Agriculture, Taking the Road of Ecological Agriculture with Chinese Characteristics". On the 19th, Dr. Zhang Lijian and his delegation were invited to visit the headquarter of the United Nations Food and Agriculture Organization (FAO, Rome) and discussed with four relevant departments including the Department of South-South Cooperation (DPSS) to discuss the agricultural application of traditional Chinese medicine principles and techniques (TCM agriculture) and reached consensus on the project cooperation. On September 28, 2019, Dr. Zhang Lijian, the initiator of "TCM Agriculture", was invited to attend the UN Industrial Development Organization (UNIDO) Global Science and Technology Innovation Conference in Shanghai, and won the Outstanding Contribution Award and delivered a special report of "TCM Agriculture Help Green Agriculture Innovation and Development" at the conference.
Academician Yuan Longping, the winner of the National Supreme Science and Technology Award and the father of Hybrid Rice, said: "This is a good and big project. I can be your consultant, and my 19 bases can be experimental fields". Li Zhensheng, the winner of the National Academy of Science and Technology Award and former vice president of the Chinese Academy of Sciences, said: "The researchers at the Chinese Academy of Sciences have been doing the relevant research. Yours are higher, more systematic and more meaningful than ecological agriculture.

On September 28, 2019, the United Nations Industrial Development Organization (UNIDO) Global Science and Technology Innovation Conference was held in Shanghai. Dr. Zhang Lijian, former vice president of the Chinese Academy of Agricultural Sciences, was invited to deliver a special report on "TCM Agriculture Help Green Agriculture Innovation and Development" at the conference, and won the "Outstanding Contribution Award". At the conference, Zhang Lijian was recommended as the executive chairman of the Global Scientific and Technological Innovation Expert Committee of the United Nations Industrial Development Organization. During the period, Academician Wang Weiqi, the chairman of the UNIDO evaluation committee of global conference on science and technology innovation award, and academician Huang Chongqi, the chairman of review supervision committee and two committee experts said that the agricultural application of traditional Chinese medicine principles and techniques (TCM agriculture) is a major innovation, conducive to agricultural sustainable development, national sustainable development, and will even benefit human and global sustainable development.

\section{Thoughts and Suggestions on "Traditional Chinese Medicine Agriculture Helping Rural Revitalization"}

(I) 1 . We should link the development of TCM agricultural industry with the strategies of rural revitalization and healthy China, and take TCM agricultural characteristic industries as the platform and important starting point to promote rural revitalization, especially rural industrial revitalization. 2. We should adhere to the principle of market leading and government support, and support the select the TCM agriculture-related leading industries with good foundation, large scale and distinctive comparative advantages in the region, to build a batch of TCM agriculture-related characteristic industrial clusters with reasonable structure and complete chains, stepping up efforts to strengthen, complement and extend the chain. 3. We should form joint efforts in agricultural development of 
traditional Chinese medicine, overcome difficulties, and form innovative development approaches and ideas. It is necessary to further excavate the characteristic resources of TCM agriculture and highlight its industrial characteristics. Efforts should be made to establish a group of different types of traditional Chinese medicine agricultural demonstration bases with obvious innovation efficiency, remarkable industry driving effect and efficient synergistic mechanism. 4. At present, we should implant "TCM agriculture" in the process of organic agriculture production, integrating the advantages, to fill the shortage of high production cost and low yield of organic agriculture, to ensure the quantity and quality of high-quality agricultural products and market demand, and to effectively promote the development of organic agriculture. 5. As the food with the highest safety coefficient, the products of traditional Chinese medicine agriculture have broad market demand, and at the same time, the production process of "TCM agriculture" emphasizes the harmony between man and nature, advocates environmental protection and ecological balance, and emphasizes sustainable development, which is a good model for the concept of "green development." National development plan of TCM agriculture should be formulated to guide and promote its healthy and orderly development. 6 . We should formulate industrial standards for TCM agriculture and build a unified certification and supervision platform. It is necessary to formulate the production standards and product standards of TCM agriculture, and special institutions should be set up to supervise the production, logistics, processing, sales and testing of TCM agriculture. We should strictly enforce product certification standards and procedures, build a unified product certification platform and traceability system, and standardize the punishment and exit mechanism. 7. We should promote multidisciplinary and collaborative research, to promote the construction of the discipline system of TCM agriculture in universities and related research institutes, to deepen the research on the key fields and mechanism of TCM agriculture, and to cultivate reserve talents. 8 . We should combine with the "double reduction" measures of chemical fertilizer of the Ministry of Agriculture and Rural Affairs, and carry out the "replacing the chemical fertilizer with the Traditional Chinese medicine fertilizer" action nationwide. We should vigorously develop Chinese herbal medicine, greatly increase the supply of Chinese herbal medicine without occupying cultivated land, making TCM agriculture fertilizer medicine, facility vegetables and fruit, emphasizing regional key points, focusing on superior producing areas, relying on the base of the park and taking the new agricultural operation subject as the core, promoting the action of replacing chemical fertilizer with agricultural fertilizer of traditional Chinese medicine to the direction of socialization and industrialization. 9. We should intensify the financial support of agricultural development of traditional Chinese medicine, give full play to the role of agricultural special funds, give priority to traditional Chinese medicine agricultural projects, set up purchase subsidy policy for traditional Chinese medicine agricultural fertilizer to the farmers and enterprises engaged in agricultural production of traditional Chinese medicine, and encourage and support agricultural fertilizer of traditional Chinese medicine research and development institutions and production enterprises. 10 . We should actively meet the social needs of health care, cultivate the industrial chain of "TCM agriculture", and promote the formation of industrial clusters in the key areas of general concern. 11. We need to establish a national experimental area of TCM agriculture to form a variety of replicable and popularizable models. At present, agricultural experimental bases of traditional Chinese medicine distributed throughout the country use Chinese herbal medicine fertilizer, organic manure, beneficial microorganisms, and marine organisms and trace fertilizers in minerals to replace chemical fertilizers, forming an efficient ecological model that can solve the problem of low yield of organic agriculture. It has led a number of agricultural enterprises nationwide, and can be upgraded to a national pilot area. 12. We will actively build the "TCM Agriculture Research and Development Platform (Center)" and develop it into a new agriculture-related discipline. TCM agriculture will add a series of new innovative highlights to the field of agricultural science and technology research and development, with the characteristics of major agricultural science and technology projects. Support should be approved at the national level. 13. We should prepare for the achievement transformation and technology integration demonstration base. The goal of the base is to produce "food homologous medicine" or "functional agricultural products" that are conducive to the sub-health population that account for about $70 \%$ of the total population to improve their immunity. We should link TCM agriculture to agricultural project bases, combining with related modern agricultural industrial park and storage and transportation of agricultural industry, to promote the connection of TCM agricultural ecological food materials and ecological restaurants; 14 . We actively create conditions, organize and set up the "Traditional Chinese Medicine Agriculture Association." We should publish 100 Cases of Agricultural Application of Traditional Chinese Medicine Principles and Techniques (TCM Agriculture) as soon as possible, and cultivate a large number of new professional farmers who are skilled in the production skills of TCM 
agriculture. 15. There are many ethnic minorities in the western region. We should increase support for the development of ethnic minority areas, and based on the cultivation of local agricultural characteristic resources, we should expand the poverty alleviation industry, extend the industrial chain, improve the mechanism of connecting interests, and make contributions to all ethnic groups into a well-off society. 16. We should establish an international cooperation platform and establish an international alliance of TCM agricultural science and technology innovation and industrial development as soon as possible. The establishment of the alliance will be conducive to summarize these experience, promote the better, more stable and faster development of TCM agriculture, expand the cooperation and exchanges of experts and related enterprises in the field and promote the theoretical and technological innovation of TCM agriculture, exploring a new way of ecological agricultural production with Chinese characteristics, and making due contributions to the construction of "community with a shared future for mankind".

(II) We should make up for the shortcomings of the "TCM agriculture", strengthen the "TCM agriculture" in basic research and development, excellent input used areas, industrial (supply) chain establishment and industrial cluster formation, farmers' interests and brand market promotion, so as to make TCM agriculture develop well and rapidly, and make greater contributions to rural revitalization and healthy China.

First, we should build a research and development platform. From the national level, we should pay close attention to resource integration, cross-border integration, complementary advantages, and integrated innovation, actively build the "TCM Agriculture Research and Development Platform (Center)", carry out systematic, in-depth and meticulous research, and develop the "TCM agriculture" into a new discipline related to agriculture.

Second, we should plan and approve projects. Traditional Chinese medicine agriculture will add a series of new innovative highlights to the field of agricultural science and technology research and development, with the characteristics of major agricultural science and technology projects. It is suggested to plan the project from the national level. A basic research and development project of cultivating efficient and multi-effective new medicinal plants (products) species to lay a good foundation for the good, fast and stable research and development of "TCM agriculture".

Third, we should prepare the achievement transformation and technology integration demonstration bases. It is suggested to build a number of demonstration bases of TCM agriculture as soon as possible to help the achievement transformation of "food homologous medicine industry development". The goals of the bases are to produce "food homologous medicine" or "functional agricultural products" that are conducive to the immunity of the sub-health population accounting for about $70 \%$ of the total population.

Fourth, we should link TCM agriculture to all levels of agricultural project bases. It is suggested to give priority to the ecological origins and the hometown of traditional Chinese medicine agriculture production bases. The modern agricultural industrial park, closely combined with the development of TCM agricultural industry, and the storage, transportation and marketing projects supporting the agricultural industry, promote the connection of TCM agricultural ecological food materials and ecological restaurants.

Fifth, we will strengthen institutional improvement and team building, actively create conditions, organize and set up the "Traditional Chinese Medicine Agriculture Association". "The Principles and Application of Traditional Chinese Medicine Agriculture" should be compiled as soon as possible, and a large number of new professional farmers who can master the production skills of TCM agriculture should be educated and trained.

Sixth, we should establish youth experience bases of traditional Chinese medicine agricultural cultivation farms, utilizing China modern agriculture and industrialization forum and platform to build a farming pastoral complex integrating with farming law, labor education, ecological catering, ecological experience, outdoor expansion, wedding photography, leisure fishing, science research and rural health, improve and maintain a high level of organic matter content of soil based on the cycle and balance of organic farming and by means of farmland waste returning to the field. Under the premise of ensuring the soil water and fertilizer conservation capacity, the soil sustainable production and utilization capacity will be gradually improved. We will adhere to the harmonious coexistence between man and nature and take the path of ecological development. We will build a platform carrier and important starting point for the revitalization of "Chinese food landmark" and "China cultural and tourism landmark" cultivation and cultural publicity and education base industry."

\section{Main Action of "Traditional Chinese Medicine Agriculture Helping Rural Revitalization" during the 14th Five-Year Plan Period}

The 14th Five-Year Plan is the first five years to start a new journey of comprehensively building a modern socialist country. It is also a key five years to comprehensively promote rural revitalization and accelerate agricultural and rural modernization. It is a milestone in China's 
development process. At present, there are 1 million new agricultural business entities in China, 2.2 million farmers cooperatives, 893,000 various agricultural socialized service organizations, and nearly 90,000 leading agricultural industrialization enterprises above the county level, creating the foundation and conditions for "agricultural application of traditional Chinese medicine principles and techniques (TCM agriculture)." During the 14th Five-Year Plan period, "Traditional Chinese Medicine Agriculture" usher in an important historical period of development and will make due contributions to rural revitalization.

I. Implant the "agricultural application of traditional Chinese medicine principles and techniques (TCM agriculture)" into the agricultural production procedures of state supported agricultural key projects, to produce products with high quality and high yield, ecological safety, good color, flavor and function, long and good resistance, and reduce production costs while improving farmland quality to achieve resource integration, complementary advantages, integrated innovation, cooperation and mutual benefits.

II. Set up a batch of "TCM Agricultural Planting and Breeding Three-dimensional Production Demonstration Base" projects, according to the "agricultural application of traditional Chinese medicine principles and techniques (TCM agriculture)" thinking and "Overall View of Traditional Chinese Medicine" design project plan.

III. Explore the establishment of a "TCM agricultural production trusteeship service system" in line with the national conditions. At present, the family operation of small and medium-sized farmers is still the most main management mode of agriculture in China. According to data, the number of small farmer households in the country accounts for $98.1 \%$ of agricultural farmers, and the area of small farmers accounts for more than $70 \%$ of the total arable land. Due to the different agricultural resource endowment, it is crucial to strengthen the role of supporting small farmers in the development of TCM agricultural industry according to local conditions. To accelerate the development of the TCM agricultural industry, we should enable small farmers to stimulate the endogenous development vitality, enhance the endogenous development driving force, enhance the endogenous development capacity, and take the development road of the endogenous TCM agricultural industry.

IV. Build the agricultural industrial chain operation mode of "market driving leading enterprises, the leading enterprises driving the bases and the bases connecting farmers" of TCM agriculture. Do a good job in both ends of the value chain of TCM agricultural industry: technology and brand, other links to integrate the best industrial chain resources, and become the organizer and integrator of the industrial chain. Form a TCM agricultural industrial system of "Industrial Park plus Logistics Park plus Service Base plus Regional Agriculture" (forming an industrial cluster-driven type).

V. Need to pay attention to two effects: 1 . The TCM agricultural industry should help to meet the requirements for the development of emerging industries in the national strategies of the Guangdong-Hong Kong-Macao Greater Bay Area, the Yangtze River Economic Belt, the Yangtze River Delta regional integration, and the coordinated development of the Beijing-Tianjin-Hebei region. 2. With the expansion of the industrial scale, the industrial layout should achieve the comprehensive improvement of the competitiveness of the whole TCM agricultural industry by promoting the development of key clusters in key areas.

VI. Expand agricultural functions, deeply integrate the development of traditional Chinese medicine agricultural industry with culture, tourism, health care and other industries, and form an emerging agricultural industry chain such as leisure agriculture. According to the data from 2019 , the number of domestic tourists is 6.006 billion, with the total tourism revenue of 6.63 trillion yuan.

VII. Implement and timely promote "Zhongguancun Green Valley Ecological Agriculture Industry Alliance" approved and issued Group standards: Agricultural Standardization of Traditional Chinese Medicine, Part 1: Standards for Production, Processing, Identification and Management, the standard number is T/GVEAIA 015-2020.

VIII. 100 Cases of Agricultural Application of Traditional Chinese Medicine Principles and Techniques (TCM Agriculture) compiled by the Branch of TCM and agriculture, WFCMS, and the International Union of TCM Agriculture will be published and distributed by China Agricultural Science and Technology Publishing House.

IX. Develop the "basic formula table of TCM agricultural inputs" as soon as possible, so that local agricultural science and technology personnel can adapt according to the local actual needs (different agricultural products and producing environment, etc.) to achieve better results in production practice, and then explore the methods of "AI matching and screening platform technology," "remote hybrid" and "haploid breeding" to form an upgraded version of the "basic matching table" with better TCM production results.

$\mathrm{X}$. Give full play to the role of TCM agriculture in the effective connection between poverty alleviation and rural revitalization industries. Over the years, the "TCM agriculture" team in the rocky desertification area in the southwest, the eastern saline area, the western cold dry 
area and the vast grassland area will implant the TCM agriculture into the local traditional agricultural production mode, forming a lot of experience and results of promotion modes of TCM agriculture technology integration in the above areas of, and based on these experience and achievements, develop the TCM agricultural industry with local characteristics, and promote the coordinated and sustainable development of social economy and ecology in the above-mentioned regions.

XI. Increase the development support for the western region, based on the local TCM agricultural characteristics (such as: complex and changeable southwest mountainous terrain preserves rich agricultural germplasm resources), cultivate and expand the TCM agricultural industry, extend the industrial chain and improve the interest linkage mechanism (the demand will appear more urgent in the "post-poverty era"), to contribute to the common progress of all ethnic groups in the western region.

\section{References}

[1] [People's Daily] "Traditional Chinese Medicine Ag- ricultural Demonstration Base" Helps Rural Revitalization http://www.caas.cn/xwzx/mtbd/306211.html.

[2] [People's Daily Online] to develop "Traditional Chinese Medicine Agriculture"-- Taking the Road of Ecological Agriculture with Chinese Characteristics http://yuqing.people.com.cn/n1/2017/0511/c21011729269394.html?

[3] [Xinhua News Agency] Economic Information Daily (Economic Information Network) http://www.jjckb. cn/2018-02/12/c_1 36969567.htm?

[4] [CPPCC Network] "Traditional Chinese Medicine Agriculture" Helps China's Agricultural Green Development http://www.caas.cn/xwzx/mtbd/3019 45.html, etc.

[5] Zhang Lijian, Wang Daolong, Liu Ruofan, Exploration and Practice of Traditional Chinese Medicine Agriculture. China Agricultural Science and Technology Publishing House, 2018. 